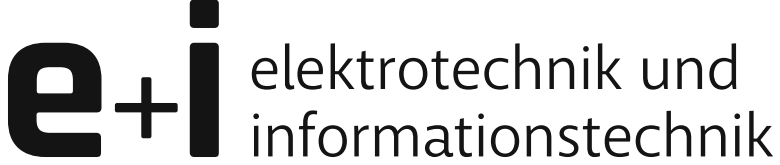

\section{Satellite and space technology}

\author{
Wilfried Gappmair
}

Accepted: 18 February 2022 / Published online: 28 February 2022

(C) The Author(s), under exclusive licence to Österreichischer Verband für Elektrotechnik (OVE) 2022

Compared to terrestrial systems, satellite-based solutions are primarily characterized by the fact that services can be provided at equal costs to all users within the coverage zone and that they form a key element for ubiquitous access on a worldwide level. Only via a satellite-based infrastructure one may connect isolated regions, like polar or offshore areas, to national and international networks under reasonable conditions. On top of that, one could observe an increasing commercialization of the low-earth orbit during the past two decades. In this context, small satellites represent an attractive option, since missions focusing on a particular objective can be realized rather quickly and in a most efficient manner-not only for communication purposes, but also with respect to navigation and earth observation.

With the goal to observe the brightness variations of massive luminous stars, the first Austrian satellite, a nano-satellite baptized BRITE-Austria/TUGSAT-1, was launched with an Indian space rocket in February 2013. In the first contribution to this special issue on satellite and space technology, Manuela Wenger and Rainer Kuschnig-both forming part of the project team from the beginning - summarize the highlights of this prominent activity, such as the discovery of a nova in the Carina constellation. It is to be noted that the mission was planned to last only two years, but after nearly nine years in space, useful data are still collected at the control center in Graz and delivered to astronomers in Austria, Canada, and Poland.

In the second paper, Reinhard Zeif and his coauthors describe a triple CubeSat developed in the framework of a project guided by the European Space Agency (ESA). Denoted as OPS-SAT, this small satellite was launched from Kourou in December 2019 with a Russian Soyuz rocket to a sun-synchronous orbit at an altitude of $515 \mathrm{~km}$. It should be verified that a reconfigurable laboratory for communication experiments can be operated in a cost-efficient way by means of commercial off-the-shelf components instead of expensive space-proof elements. In the third contribution, which is also connected to OPS-SAT, Manuel Kubicka et al. describe the design and test of the thermal management such as it is necessary for this kind of satellites. In this respect, useful results were achieved, which are most helpful for future missions.

The third satellite, a triple CubeSat named PRETTY, which aims to measure the elevation and roughness of ice surfaces in polar regions, is still under construction. This satellite is being developed by a consortium consisting of TU Graz, RUAG Space, and Seibersdorf Laboratories in the framework of an ESA project. In their paper, Andreas Dielacher and his co-authors describe both the concept and platform employed for earth observation. Part of this project is also the fifth paper by Andreas Hörmer et al., which focuses on the implementation and test of a fault-tolerant power supply for the PRETTY spacecraft. A considerable portion of small satellites fail within the first year of operation, and it turned out that the power supply is the main reason for this problem. 


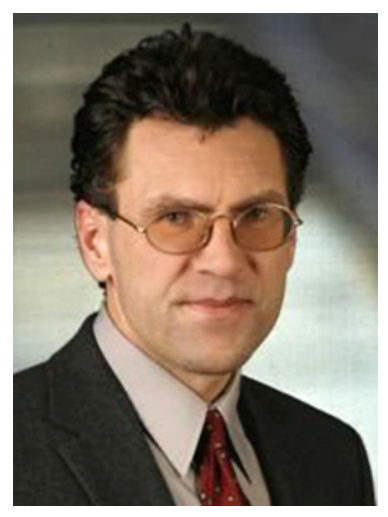

Wilfried Gappmair, Wilfried Gappmair's main interests and researchactivitiesarefocusedon communication theory, channel coding, digital modulation, parameter estimation, synchronization in digital receivers, as well as satellite and optical wireless communications. $\mathrm{He}$ is the author and co-author of about 90 refereed journal and conference papers covering his areas of interest, he contributed articles to four books, and he holds four patents. Furthermore, he is a Senior Member of the Institute of Electrical and Electronics Engineers (IEEE), Member of the Institution of Engineering and Technology (IET), and Member of the Austrian Electrotechnical Association (OVE). 